möchte ich nur an das Grundlagenwerk von Waldfried Pechtl (1989), der in seinem Buch „Zwischen Organismus und Organisation“ einen für die damalige Zeit vielleicht viel zu frühen Diskurs angestoßen hat. Dieser Diskurs wird heute in der DÖK weitergeführt und im Unterschied zu den meisten BA-Instituten in einer bemerkenswerten Weise konzeptionell, aber auch praxeologisch weiterentwickelt. Integriert werden dabei, wie im Beitrag von Schenk deutlich wird, die Bezugnahme auf psychoanalytische Organisationstheorien, Sozialwissenschaften und systemische Überlegungen (z. B. Luhmann). Organisationen werden dabei u.a. als Energiefelder betrachtet, in denen sich einzelne Personen als Funktionsträgerinnen, Gruppierungen und Teams, Abteilungen und Teilorganisationen, aber auch die Kundinnen, z.B. betreute Menschen, formell und informell bewegen. „Niemand kann unabhängig von diesem Feld handeln und gleichzeitig trägt jede Person zum Energiefeld bei“ (S. 283). Schenk entwickelt hierauf aufbauend in Bezug auf das Sechs-Felder-Modell von Marvin Weisbord (1984) ein spezifisches Modell, das durch einen relationalen Ansatz besticht. So geht es in dem Modell u.a. um die Inter- dependenzen von Teilen der Organisation, wie Ziele, Strukturen, Beziehungen, Belohnungen, Führung usw., sowie um Entwicklung von Prozesskompetenz in einer solchen Organisation.

Es ist wahrscheinlich nicht zu übersehen, dass ich fasziniert bin von dem Buch und am liebsten noch detaillierter fortfahren möchte. Meine Buchbesprechung mag daher auch als Appell verstanden werden, die BA sowie körperpsychotherapeutische Zugänge zu betrachten und weiterzuentwickeln im Sinne von gesellschaftspolitischem Handeln. Dürfen wir als KörperpsychotherapeutInnen doch nicht nur warten, bis jemand mit einer wie auch immer schweren Symptomatik in der eigenen psychotherapeutischen Praxis auftaucht. Besinnen wir uns auf einen solch möglichen Selbst-Appell, arbeiten wir auch im Sinne von Wilhelm Reich oder anderen körperpsychotherapeutischen Pionieren. Hieß es in den 1980er Jahren, angestoßen durch Alexander Lowen, „back to the roots“, so könnte die Arbeit jetzt verstanden werden als „back to the people“ oder „back to the humans as part of society“.

Dipl. rer. soc. Ulrich Sollmann

DOI 10.2378/ktb2020.art23d

\title{
David Schnarch: Brain Talk. Wie wir das Gehirn nutzen, um uns selbst und andere besser zu verstehen
}

\section{Kösel Verlag, 2020, München, 587 Seiten, 30,00€ (D)}

ch hatte keinen Sinn mehr darin gesehen, و zum x-ten Mal auf die Matte einzuprügeln und meine Wut auf meine Mutter auszudrücken“, höre ich gelegentlich von KlientInnen als Antwort auf meine Frage, wieso sie ihre vorige Körperpsychotherapie abgebrochen haben. Die Körperpsychotherapie ist stark, wenn es darum geht, emotionale Prozesse in Menschen wahrzunehmen und zu lenken. Für das Verstehen der
Komplexität menschlicher Interaktionen und ihrer psychischen Auswirkungen hat die Kör-

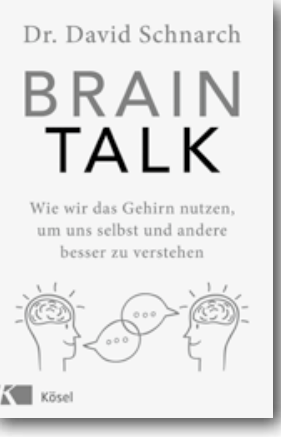
perpsychotherapie traditionell weniger viel zu bieten. Da psychische Veränderungen vielfach als Folge energetischer und emotionaler Prozesse verstanden werden, heißt es: Free your ass, your mind will follow! 
In meiner eigenen neoreichianischen Ausbildung wurden die kognitiven, verbalen, und relationalen Aspekte der Therapie etwas stiefmütterlich behandelt. „Was spürst du gerade in deinem Körper?“ ist sicherlich oft eine hilfreiche Frage. Ebenso oft reicht sie aber auch nicht. Es mag zwar erleichternd sein, wenn ich meine Mutter/meinen Vater symbolisch beschimpfen und zusammenschlagen kann, doch das ist bestenfalls der erste Schritt, eine erwachsene Beziehung zu ihnen und ihren Botschaften zu entwickeln. Seit einiger Zeit gibt es daher Bestrebungen, die Körperpsychotherapie mit Elementen aus anderen Verfahren und Forschungen wie Psychoanalyse, Bindungsforschung und die interpersonelle Dimension anzureichern.

Eine solche Bereicherung für unser Feld ist David Schnarchs neuestes Buch „Brain Talk Wie wir das Gehirn nutzen, um uns selbst und andere besser zu verstehen“. Es bietet einen neuen Blick auf das Verstehen zwischenmenschlicher Interaktionen, auf die daraus erwachsenden Traumata und wie sie sich behandeln lassen.

Im Zentrum von „Brain Talk“ steht die Erkenntnis, dass Menschen sich in sozialen Situationen stets ein Bild vom inneren Zustand ihres Gegenübers machen. Diese Fähigkeit wird seit den späten 1970er Jahren erforscht und ist unter verschiedenen Namen bekannt: „Theory of Mind“, „Mentalisierung“ oder „soziale Kognition“ (die Begriffe überlappen sich, sind aber nicht völlig synonym). David Schnarch nennt es „Mindmapping“. Schnarch hat sich als Innovator der Paar- und Sexualtherapie international einen Namen gemacht. Wieso er sich nun mit Mindmapping, Hirnforschung und interpersonellen Traumatisierungen beschäftigt, erscheint auf den ersten Blick erklärungsbedürftig. Tatsächlich aber spielte die Fähigkeit, andere Menschen „lesen“ zu können, in seiner therapeutischen Arbeit mit Paaren schon lange eine zentrale Rolle. In „Brain Talk“ führt er aus, wie das Mindmap- ping sowohl zum Wohle als auch zum Schaden anderer Menschen eingesetzt wird und welche schwerwiegenden, im Gehirn nachweisbaren Traumatisierungen es erzeugen kann.

Schnarch zeigt auf, wie Empathie auch antisozial eingesetzt werden kann. Wir benötigen Einfühlungsvermögen, um unseren Partner mit einem Geschenk zu überraschen, aber auch, wenn wir ein falsches Bild von uns entwerfen wollen, wenn wir lügen und betrügen. Der deutsche Begriff "Schadenfreude“ beschreibt dabei einen der harmloseren Aspekte der antisozialen Empathie. Wir können den Schaden eines anderen schließlich nur dann genießen, wenn wir eine Vorstellung davon haben, was dieser Mensch fühlt. Sarkasmus wäre ein weiteres Beispiel für antisoziale Empathie.

Schnarch widerspricht der Annahme des Mentalisierungskonzeptes, dass die Entwicklung der Mentalisierungsfähigkeit die empathische Spiegelung durch Bindungspersonen in der Kindheit voraussetzt. Im Gegenteil, lernten gerade Kinder aus gewalttätigen und missbräuchlichen Elternhäusern, „die Zeichen zu lesen“. Nicht alle Eltern, so Schnarch weiter, würden das Beste für ihre Kinder wollen. Manche Eltern quälen und demütigen ihre Kinder nicht etwa, weil sie nicht ahnen würden, was das bei ihnen anrichtet, sondern eben weil sie es sehen können. Schnarch sieht in der weitgehenden Weigerung der Psychotherapie, diesem Fakt Rechnung zu tragen, einen schwerwiegenden blinden Fleck (nicht nur) in unserer Profession: „Aber wir ziehen vor, das, was wir heimlich wissen und nicht zugeben wollen, abzustreiten: Grausam zu sein kann sich gut anfühlen. Das ist der Grund, warum Menschen es tun und warum ihre Gedanken und Handlungen besonders ekelhaft sind, wenn sie es tun.“ (S. 199)

Schnarch zeigt anhand von Fallbeispielen, wie weit verbreitet seelische Grausamkeit in Familien - auch und gerade in den Familien der Mittel- und Oberschicht - ist und welche 
Folgen dies für das Gehirn, die seelische Gesundheit und menschliche Beziehungen hat.

„Traumatisches Mindmapping“, ein weiterer von Schnarch geprägter Begriff, entsteht dann, wenn wir im Geist unseres Gegenübers etwas lesen, das uns zutiefst verstört - wenn wir erkennen, dass hinter dem Verhalten der Mutter keine Überängstlichkeit steckt, sondern der Wunsch zu demütigen; wenn die Tochter in den Augen ihres Vaters erkennt, dass er sie sexuell begehrt; oder wenn die Realität eines Kindes von einem Elternteil massiv verdreht wird: „Ich habe dir nie versprochen, dass du einen kleinen Hund bekommst, wenn du Klassenbester wirst!“ (auch als „Gaslighting“ bekannt, benannt nach dem Film mit Ingrid Bergman).

Traumatisches Mindmapping zeigt sich später in einer Vielzahl von Symptomen, wie Depressionen, Ängste, Selbstzweifel, Denkstörungen, Dissoziation, Lücken im autobiografischen Gedächtnis und eine in bestimmten Situationen eingeschränkte Fähigkeit, jemanden korrekt „lesen“ zu können (Schnarch nennt das „Spaghettihirn“). Er diskutiert im Anhang, wie sich diese Symptome im Rahmen der Komplexen Posttraumatischen Belastungsstörung (K-PTBS) verstehen lassen.

Glücklicherweise belässt es Schnarch nicht beim Blick in menschliche Abgründe, er führt auch aus, wie wir diese Beziehungstraumata behandeln können. Mit den von ihm entwickelten schriftlichen Dialogen mit dem Antagonisten (wie Eltern, Partner, Chef oder einer abwertenden innere Stimme), bringt man sich selbst dazu, dem eigenen traumatischen Mindmapping ins Antlitz zu blicken. Dabei geht es erst in zweiter Linie um eine Konfron- tation des Antagonisten. Im Vordergrund stehen die Konfrontation und die Überwindung der in unseren Köpfen nistenden Lügen über uns selbst und über die Welt - Lügen, die uns daran hindern, uns zu unserer vollen Größe aufzurichten.

Anders als es der etwas nichtssagende Titel vermuten lässt, nimmt uns „Brain Talk“ mit auf eine ebenso fesselnde wie erschütternde Reise durch bislang von der Psychotherapie nur ansatzweise verstandenen Traumatisierungen. Es ist auch in der deutschen Version flüssig und gut lesbar geschrieben und richtet sich sowohl an interessierte Laien als auch an therapeutisch und wissenschaftlich Tätige. Das Buch liefert praktische Anleitung zur Selbsterforschung, verknüpft klinische Beobachtungen mit der Forschung, und diejenigen, die tiefer einsteigen wollen, finden einen über einhundertseitigen wissenschaftlichen Anhang.

Die Einführung von Mindmapping in die (Körper-)Psychotherapie hilft uns, das therapeutische Vorgehen passgenauer und ökonomischer zu gestalten. Wir bekommen zudem ein Mittel an die Hand, mit dem wir Realitätskonstruktionen unserer KlientInnen überprüfen können, was uns andere Möglichkeiten der Intervention und Konfrontation bietet.

Das Buch ist ein Augenöffner für jeden, der menschliche Beziehungen besser verstehen möchte. In Zeiten von „alternativen Fakten“ und anderen Versuchen, unsere Realität zu manipulieren, bietet dieses Buch ein potentes Gegengift.

Marc Rackelmann

DOI 10.2378/ktb2020.art24d 